\title{
Synthèse, étude toxicologique et activité psychotrope de nouvelles arylidènpyridazin- 3-ones N-substituées
}

Adnane BENMOUSSA ${ }^{1 *}$, Pascal Manuele KANYONGA',3, M'Hammed ANSAR ${ }^{1}$, Amina ZELLOU2, J EL HARTI' ${ }^{1}$, Jamal LAMSAOURI ${ }^{1}$, My Abbes FAOUZI ${ }^{2}$, Ahmed ZAHIDI ${ }^{1}$, Hamid BENZIANE'1, Yahia CHERRAH² \& Jamal TAOUFIK1

1: Laboratoire de Chimie Thérapeutique, Faculté de Médecine et de Pharmacie, Université Mohamed V-Souissi, BP 6203, Rabat Instituts, Rabat-Maroc

2: Laboratoire de Pharmacologie et de Toxicologie, Faculté de Médecine et de Pharmacie, Université Mohamed VSouissi, BP 6203, Rabat Instituts, Rabat-Maroc

3: UER de Biochimie, Chimie médicale et pharmacologie, I.S.T.M-Kinshasa/ R.D. Congo.

\begin{abstract}
RESUME
Les dérivés oxygénés de la pyridazine sont connus en thérapeutique pour la richesse de leurs potentiels pharmacologiques : antihypertenseur et cardiotonique. Des propriétés antibactériennes sont également mentionnées dans la littérature.

De ces considérations, résulte l'idée de synthétiser et d'étudier de nouvelles séries d'arylidènpyridazin-3-ones substituées sur le plan toxicologique, et d'explorer leur activité psychotrope.

L'étude de l'activité pharmacologique a démontré que ces produits ne sont pas toxiques aux doses thérapeutiques et qu'ils sont doués d'effets sédatif, myorelaxant, anxiolytique mais, ne présentent pas d'effet hypnotique, ni cataleptique. Cependant, ces trois produits de synthèse potentialisent à des degrés différents l'effet hypnotique du diazepam. Mots clefs : arylidènpyridazin-3-ones N-substituées, synthèse, toxicité aigue, activité psychotrope.
\end{abstract}

\section{ABSTRACT}

Synthesis, toxicological study and psychotropic activity of new arylidenpyridazin-3-ones $\mathrm{N}$-substituted.

The oxygenated derivatives of pyridazin are known into therapeutic for the richness of their pharmacological potentials: antihypertensive and cardiotonic. Properties antibacteriennes are also mentioned in the literature. From these considerations, results the idea to synthesize and study new series of arylidenpyridazin-3-ones $\mathrm{N}$-substituted on the toxicological level, but also, to explore their possible activity on the central nervous system, in particular the psychotropic activity.

The study of the pharmacological activity has shown that these products are not toxic at therapeutic doses and possess sedative, muscle relaxant, anxiolytic effects, but do not present any hypnotic, or catalepsy effect. However, these three products potentiate synthesis to different degrees of the hypnotic effect diazepam.

Key words: arylidenpyridazin-3-ones N-substituted, synthesis, acute toxicity, psychotropic activity.

\section{INTRODUCTION}

L'hétérocycle de la pyridazinone est connu en thérapeutique pour la richesse de ses potentiels pharmacologiques: antihypertenseur, cardiotonique et analgésique. Et actuellement certains auteurs ont mis en évidence les propriétés antibactériennes de ces composés [13]. Cependant, en plus de ces activités pharmacologiques, ces composés présentent un intérêt sur le plan de la réactivité, c'est ainsi que plusieurs travaux ont montré que la substitution de la pyridazinone au niveau de l'azote par un agent alkylant lui confère des propriétés pharmacologiques très intéressantes [4, 5], et d'autres ont démontré que les dérivés alkylés par un radical aromatique, halogéné ou méthoxy peuvent se révéler plus réactifs que les autres produits $\mathrm{N}$ - substitués $[6,7,8]$ et d'autres auteurs ont démontré que ces produits présentent une action sédative remarquable et que certain substituant les rendaient moins toxiques $[6,7]$ C'est ainsi que dans le cadre de nos recherches dans la série de pyridazinones, nous nous sommes intéressés à la synthèse des produits $\mathrm{N}$ substitués et à l'évaluation leur activité pharmacologique sur le système nerveux central, grâce à une série de tests comportementaux employés en psychopharmacie, en comparaison avec un produit de référence. 
MATERIEL ET METHODES

Synthese des produits.

La synthèse des acide3-arylidènelévuliniques se produit par une réaction de condensation à partir de l'acide lévulinique des aldéhydes aromatiques
[9]. Les acides ainsi obtenus ont été transformés par réaction d'addition nucléophile d'hydrate d'hydrazine, en 5-arylidèn-6-méthylpyridazin-3ones (Figure 1)<smiles>[R]c1ccc(C=O)c([R2])c1</smiles><smiles>[R]c1ccc(/C=C(\CC(=O)O)C(C)=O)c([R2])c1</smiles><smiles>[R]c1ccc(/C=C2/CC(=O)NN=C2C)c([R])c1</smiles>

Figure 1

L'iodure d'éthyle, par une attaque nucléophile du carbone déficitaire en électrons des 5-arylidèn-6méthylpyridazin-3-ones, permet d'accéder aux 5-arylidèn-2-éthyl-6-méthylpyridazin-3-ones (Figure 2).<smiles>[R]c1ccc(Cl)c([R2])c1</smiles><smiles>C=C1CC(=O)N(C(C)C(=O)O)N=C1C</smiles><smiles>[R]c1ccc(/C=C2/CC(=O)N(CC)N=C2C)c([R2])c1</smiles>

$\begin{array}{lll} & \mathrm{R}_{1} & \\ \text { a } & & \mathrm{H} \\ \text { b } & & \mathrm{OCH}_{3} \\ & & \mathrm{H} \\ \text { d } & & \mathrm{NO}_{2} \\ & & \mathrm{Cl}\end{array}$

$\mathrm{R}_{2}$

$$
\begin{aligned}
& \mathrm{H} \\
& \mathrm{H} \\
& \mathrm{Cl} \\
& \mathrm{H} \\
& \mathrm{Cl}
\end{aligned}
$$

Figure 2 
L'obtention des produits se fait selon le mode opératoire ci-dessous :

Synthèse des 5-arylidènpyridazin-3-ones. Les acides 3-arylidènelévuliniques sont obtenus en agitant à température ambiante pendant 24 heures :

- 0,2 mole d'acide lévulinique ;

- 0,1mole d'aldéhyde aromatique en présence d'acide chlorhydrique anhydre généré à partir de $2 \mathrm{M}$ de chlorure de sodium et de $1 \mathrm{M}$ d'acide sulfurique concentré.

Le mélange réactionnel est repris par le minimum d'eau et extrait par le chlorure de méthylène. La phase organique ainsi obtenue est séchée pendant 12 heures sur le chlorure de calcium, puis évaporée à sec sous pression réduite et le produit est recristallisé dans l'éthanol.

Dans un ballon de $250 \mathrm{ml}$ ont été mélangé: 0,01moles d'acide 3-arylidènelévulinique en solution dans $30 \mathrm{ml}$ d'éthanol et 0,5 moles d'hydrate d'hydrazine. Le mélange a été porté à reflux pendant deux heures, puis après refroidissement les 5-arylidènes-6méthylpyridazine-3-ones obtenues sous forme de précipité sont recristallisées dans l'éthanol.
Synthèse des 5-arylidèn-2-éthyl-6-méthyl(2H, $4 \mathrm{H})$ pyridazin-3-ones. Dans un ballon de $250 \mathrm{ml}$ ont été mélangé: 0,05 moles d'arylidèn-6méthylpyridazinone et 0,07 moles d'hydroxyde de potassium, dans $100 \mathrm{ml}$ d'éthanol sous agitation. Après dissolution complète nous avons ajouté 0,06 mole d'iodure d'éthyle comme agent alkylant. Et, la réaction est maintenue sous agitation à la température ambiante pendant 72 heures. Le solvant est évaporé sous pression réduite, et le mélange est repris par $200 \mathrm{ml}$ d'eau. Le précipité formé est filtré, séché et recristallisé dans l'éthanol.

Les structures de ces produits ont été élucidées sur base des données spectrales et des paramètres physicochimiques (Tableaux 1, 2 et 3 ) obtenus à partir des techniques ci-après :

Le point de fusion a été déterminé à l'aide d'un appareil BUCHI SMP 20 et n'est pas corrigé. Le spectre IR a été enregistré en film de $\mathrm{KBr}$, sur un spectromètre Jasco FT/IR-460 plus. Les spectres de RMN ${ }^{1} \mathrm{H}$ ont été enregistrés sur un spectromètre BRUKER modèle AVANCE80 opérant à $300 \mathrm{MHz} \quad\left({ }^{1} \mathrm{H}\right)$. Les déplacements chimiques sont exprimés en ppm par rapport au tétraméthylsilane (TMS) utilisé comme référence ( $T M S=0)$.

Tableau 1 : Caractéristiques physicochimiques des pyridazinones

\begin{tabular}{ccc}
\hline $\mathrm{N}^{\circ}$ & Rendement $\%$ & Point de fusion en ${ }^{\circ} \mathrm{C}$ \\
\hline 3a & 80 & 174 \\
3b & 76 & 190 \\
3c & 72 & 209 \\
3d & 74 & 239 \\
3e & 70 & 220 \\
\hline
\end{tabular}

Tableau 2 : Caractéristiques physicochimiques des pyridazinones N-éthyle.

\begin{tabular}{ccc}
\hline $\mathrm{N}^{\circ}$ & Rendement $\%$ & Point de fusion en ${ }^{\circ} \mathrm{C}$ \\
\hline $4 \mathrm{a}$ & 52 & 61 \\
$4 \mathrm{~b}$ & 69 & 166 \\
$4 \mathrm{c}$ & 65 & 110 \\
$4 \mathrm{~d}$ & 54 & 95 \\
$4 \mathrm{e}$ & 50 & 80 \\
\hline
\end{tabular}


Tableau 3: Paramètres spectrales des produits testés

Produit

Données spectrales

5-(1'-benzylidène)-6-méthyl-(2H, 4H)-pyridazin-3-one

5-(4'-méthoxy-1'-benzylidène)-6méthyl-(2H, 4H)-pyridazin-3-one.

5-(1'-benzylidène)-2-éthyl-6méthyl $(2 \mathrm{H}, 4 \mathrm{H})$ pyridazin-3-one.
Spectre IR: $\quad(\mathrm{N}-\mathrm{H})=3100 \mathrm{~cm}^{-1}, \quad(\mathrm{C}=\mathrm{O})=1640 \mathrm{~cm}^{-1}$; Spectre RMN ${ }^{1} \mathrm{H}$ $\left(\mathrm{CDCl}_{3}\right) \quad 2,15\left(\mathrm{~s}, 3 \mathrm{H}, \mathrm{CH}_{3}\right), 3,8\left(\mathrm{~s}, 2 \mathrm{H}, \mathrm{CH}_{2}\right), 6,3(\mathrm{~s}, 1 \mathrm{H}, \mathrm{CH}), 7,0-7,3(\mathrm{~m}$, $5 \mathrm{H}, \mathrm{Ars}), 12,8(\mathrm{ls}, 1 \mathrm{H}, \mathrm{NH})$.

Spectre IR: $\quad(\mathrm{N}-\mathrm{H})=3100 \mathrm{~cm}^{-1}, \quad(\mathrm{C}=\mathrm{O})=1659 \mathrm{~cm}^{-1}$; Spectre RMN ${ }^{1} \mathrm{H}$ $\left(\mathrm{CDCl}_{3}\right): 2,3$ (s, 3H, $\left.\mathrm{CH}_{3}\right), 2,5$ (s, 3H, OCH $), 3,7$ (s, 2H, $\left.\mathrm{CH}_{2}\right), 6,4$ (s, $1 \mathrm{H}$, $\mathrm{CH}), 6,9-7,2$ (m, 4H, Ars), 11,5 (ls, 1H, NH).

Spectre IR: $(\mathrm{C}=0)=1660 \mathrm{~cm}^{-1}$; Spectre RMN ${ }^{1} \mathrm{H}\left(\mathrm{CDCl}_{3}\right) \quad 1,3(\mathrm{t}, 3 \mathrm{H}$, $\left.\mathrm{CH}_{2}-\mathrm{CH}_{3}\right), 2,2$ (s, 3H, CH 3 ), 3,8 (s, 2H, $\left.\mathrm{CH}_{2}\right), 4,2$ (q, 2H, $\left.\mathrm{CH}_{2}-\mathrm{CH}_{3}\right), 6,5$ (s, $1 \mathrm{H}, \mathrm{CH}), 7,0-7,4$ (m, 5H, Ars).

5-(4'-méthoxy-1'-benzylidène)-2- Spectre IR: $(\mathrm{C}=0)=1659 \mathrm{~cm}^{-1} \quad$; Spectre $\mathrm{RMN}{ }^{1} \mathrm{H}\left(\mathrm{CDCl}_{3}\right) \quad 1,3(\mathrm{t}, 3 \mathrm{H}$, éthyl-6-méthyl $(2 \mathrm{H}, 4 \mathrm{H})$ pyridazin- $\left.\mathrm{CH}_{2}-\mathrm{CH}_{3}\right), 2,3\left(\mathrm{~s}, 3 \mathrm{H}, \mathrm{CH}_{3}\right), 3,9\left(\mathrm{~s}, 2 \mathrm{H}, \mathrm{CH}_{2}\right), 4,4\left(\mathrm{q}, 2 \mathrm{H}, \mathrm{CH}_{2}-\mathrm{CH}_{3}\right), 6,7$ (s, 3-one. $1 \mathrm{H}, \mathrm{CH}), 6,8-7,2$ (m, 5H, Ars).

Les symboles suivants ont été utilisés : $\mathrm{s}=$ singulet ; $\mathrm{t}=$ triplet ; $\mathrm{m}=$ multiplet $; \mathrm{l}=$ large singulet, les constantes de couplage $\mathrm{J}$ sont exprimées en $\mathrm{Hz}$.

\section{Etude pharmacologique} Les produits testés sont :

4a : $\quad$ 5-(1'-benzylidène)-2-éthyl-6-méthyl(2H, 4H)pyridazin-3-one

4b : $\quad 5$-(4'-méthoxy-1'-benzylidène)-2-éthyl-6méthyl $(2 \mathrm{H}, 4 \mathrm{H})$ pyridazin-3-one

4c : $\quad$ 5-(2'-chloro-1'-benzylidène)-2-éthyl-6méthyl $(2 \mathrm{H}, 4 \mathrm{H})$ pyridazin-3-one.

Le produit de référence est le diazépam.

Les animaux. L'expérience est effectuée sur des souris swiss adultes et des rats wistar. Ces animaux proviennent de l'élevage du Laboratoire de Pharmacologie de la Faculté de Médecine et de Pharmacie de Rabat. Le poids des souris se situe entre 25 et $30 \mathrm{~g}$, celui des rats entre 200 et $250 \mathrm{~g}$. Ils étaient maintenus dans les mêmes conditions de température $\left(25 \pm 2^{\circ} \mathrm{C}\right)$, l'humidité relative était située entre $50 \pm 5 \%$, et l'accès à l'eau et aux aliments à volonté [10]. L'utilisation des animaux a été faite en conformité avec les guides d'utilisation des animaux de Laboratoire [11-13].

Toxicité aigüe. Elle est déterminée selon la méthode de Lichfield et wilcoxon [14], dans laquelle cinq lots de dix souris reçoivent les composés testés par deux voies distinctes:

- La voie orale sous forme de suspension dans la gomme arabique à $10 \%$. Les doses utilisées par lot des souris sont les suivantes: $200,400,800,1600$, et $2000 \mathrm{mg} / \mathrm{Kg}$.

- Parallèlement, un essai limite à la dose de $1000 \mathrm{mg} / \mathrm{Kg}$ a été réalisé avec les mêmes produits par voie intrapéritonéale sous un volume de $0,5 \mathrm{ml}$ dans le tween 80 à $5 \%$ pour $20 \mathrm{~g}$ de poids corporel de souris.

Les animaux sont gardés en observation pendant 14 jours.

\section{Activité psychotrope}

La mise en évidence de l'effet sédatif, tranquillisant, myorelaxant, hypnotique et catatonigène a été réalisée grâce aux tests comportementaux suivants $[15,16]$

- test de traction: II consiste à suspendre des souris par les pattes antérieures à un fil métallique tendu horizontalement, et on compte le temps mis par la souris pour amener au moins une des pattes postérieure à toucher le fil. Une souris normale effectue un rétablissement en moins de 5 secondes, le cas contraire signifie que la souris est soumise à une action sédative.

- test de la cheminée: II consiste à placer une souris dans un tube de verre de $30 \mathrm{~cm}$ de longueur, disposé verticalement. La réponse est positive si la souris remonte le tube en moins de 30 secondes.

- test de la planche à trous : II permet d'explorer la curiosité et le désir de fuite de l'animal. Pour ce 
faire, on utilise une planche de $40 \times 40 \mathrm{~cm}$ et de $1,8 \mathrm{~cm}$ d'épaisseur, percé de 16 trous de $3 \mathrm{~cm}$ de diamètre, régulièrement espacés. La souris est déposée au centre de la planche et on compte le nombre de fois où la souris plonge la tête dans un des trous au bout de 1, 2, 3, 4 et 5 minutes et on calcule a moyenne de trous explorés pendant 5 minutes.

- recherche d'une action catatonigène: On administre le produit par voie intra péritonéale aux rats et on suit toutes les 15 minutes l'évolution de la catalepsie. L'animal est considéré comme cataleptique, s'il accepte de croiser les pattes antérieures avec les pattes postérieures homolatérales, et le fait de garder cette position implique une catalepsie importante. On note pour chaque animal le temps d'apparition de la catalepsie.

- recherche de l'action hypnotique : les animaux reçoivent les produits par voie intra péritonéale, et on observe la perte du réflexe de redressement en plaçant les animaux sur le dos, ceux qui gardent cette position sont considérés comme hypnotisés, contrairement à ceux qui se retournent. Et on mesure alors le temps écoulé entre l'injection du produit et la suppression du réflexe de redressement, temps d'endormissement (T.E.), et le temps écoulé entre la disparition du réflexe de redressement et sa réapparition $=$ temps de sommeil (T.S.).

- étude des interactions médicamenteuses versus le thiobarbital sodique.

Pour chaque test, trois lots de cinq animaux (souris et rats) sont utilisés et l'administration se fait exclusivement par voie intrapéritonéale:

o lot témoin recevant le solvant de la drogue (le Tween 80 à $5 \%$ ).

o lot de référence recevant le diazépam à la dose de $50 \mathrm{mg} / \mathrm{Kg}$.

- lot traité par la drogue

- Les doses utilisées (des produits $4 a, 4 b$ et $4 c$ ) sont réparties de la manière suivante :

- 150 et $200 \mathrm{mg} / \mathrm{Kg}$ pour les souris,

- 200,400 et $600 \mathrm{mg} / \mathrm{Kg}$ pour les rats.

Ces doses ont été déterminées sur base des résultats de l'étude de la toxicité aigue de ces produits, nous avons considéré les doses supérieures ou égales au $1 / 10^{\text {eme }}$ de la $D_{50}$ de ces produits.

\section{RESULTATS ET DISCUSSION.}

Toxicité aiguë. Dans nos conditions expérimentales, les composés testés s'avèrent moins toxiques, avec une $D_{50}$ supérieure à 2 $\mathrm{g} / \mathrm{Kg}$ par voie orale. La dose limite à $1 \mathrm{~g} / \mathrm{Kg}$ par voie intrapéritonéale n'a entraîné aucune létalité jusqu'à 14 jours.

Activités psychotropes. Les résultats sont exprimés par rapport au lot témoin et au lot de référence.

- Test de traction: Le temps de rétablissement des souris traitées par les produits testés est nettement allongé par rapport au lot des souris témoins. En plus nous avons enregistré des chutes dans le lot des souris traitées (Tableau 4).

- Test de la cheminée: Nous avons noté une perte d'initiative et de la curiosité dans le lot des souris traitées par les produits testés (tableau $4)$ : cette action est moins prononcée pour le produit $\mathbf{4 b}$.

- Test de la planche à trous : Les produits testés surtout le 4c diminuent le nombre cumulatif de trous explorés (en rapport avec la curiosité) et le nombre d'espaces parcourus entre deux trous (en rapport avec l'activité motrice, tableau 4)

- Effet hypnotique: Aux doses de 200 et 400 $\mathrm{mg} / \mathrm{Kg}$ en intrapéritonéale, les produits testés n'ont pas induit un effet hypnotique sur les rats, mais à la dose de $600 \mathrm{mg} / \mathrm{Kg}$, l'effet hypnotique induit par ces produits était comparable à celui du produit de référence diazépam (tableau 5).

- Effet cataleptique: Les différents produits testés n'ont pas présenté un effet cataleptique chez les rats traités (Tableau 5).

- Interaction médicamenteuse: Les trois produits prolongent l'effet hypnotique du thiobarbital sodique suggérant une synergie d'action, avec un temps de sommeil supérieur pour le produit $4 c$ par rapport à la référence (Tableau 6).

L'ensemble des résultats semble indiqué que les trois produits expérimentés sont moins toxiques aux doses utilisées et exercent un effet sédatif sur le système nerveux central. Sur le comportement psychomoteur des souris, le produit $4 c$ à une activité supérieure par rapport aux deux autres produits $\mathbf{4 a}$ et $\mathbf{4 b}$.

Vis-à-vis du diazépam, les produits $4 a$ et $\mathbf{4 b}$ ont une action sédative moins importante que celle du diazépam, tandis que le produit $4 c$ a une action comparable. L'activité sur le système nerveux 
central semble augmenter avec la substitution au niveau de l'azote par un halogène comme c'est indiqué dans certains travaux $[7,8]$.
Ces résultats démontrent que les produits testés possèdent des propriétés hypnotiques très marquées à la dose utilisées.

Tableau 4 : Action sédative des produits 4a, 4b et 4c à la dose de 150 et $200 \mathrm{mg} / \mathrm{Kg}$

\begin{tabular}{|c|c|c|c|c|c|c|c|c|c|}
\hline & & \multirow{2}{*}{ Témoin } & \multirow{2}{*}{ Référence } & \multicolumn{3}{|c|}{$150 \mathrm{mg} / \mathrm{Kg}$} & \multicolumn{3}{|c|}{$200 \mathrm{mg} / \mathrm{Kg}$} \\
\hline & & & & $4 a$ & $4 b$ & $4 c$ & $4 a$ & $4 b$ & $4 c$ \\
\hline \multirow{3}{*}{ Traction } & $\begin{array}{l}\text { Nombre de } \\
\text { chutes }\end{array}$ & 0 & 5 & 2 & 3 & 4 & 3 & 3 & 4 \\
\hline & $\begin{array}{l}\text { Tm1 en } \\
\text { secondes }\end{array}$ & & $7,3 \pm 0,9$ & $10,5 \pm 0,3$ & $6,2 \pm 0,02$ & $9 \pm 1$ & $6,5 \pm 0,5$ & $4,6 \pm 0,2$ & $6,8 \pm 0,2$ \\
\hline & $\begin{array}{l}\text { Tm2 en } \\
\text { secondes }\end{array}$ & $\begin{array}{c}n=5 \\
0,5 \pm 0,1\end{array}$ & & $\begin{array}{c}n=3 \\
12 \pm 1\end{array}$ & $\begin{array}{c}n=2 \\
10 \pm 0,1\end{array}$ & $\begin{array}{c}n=2 \\
37,5 \pm 1\end{array}$ & $\begin{array}{c}n=2 \\
22 \pm 1\end{array}$ & $\begin{array}{l}n=2 \\
9 \pm 1\end{array}$ & $\begin{array}{c}n=2 \\
39,5 \pm 1\end{array}$ \\
\hline \multirow[b]{2}{*}{ Cheminée } & $\begin{array}{l}\text { Réponse } \\
\text { positive }\end{array}$ & 5 & 0 & 0 & 2 & 0 & 2 & 2 & 0 \\
\hline & $\begin{array}{l}\text { Tm pour } \\
\text { remonter en } \\
\text { minutes }\end{array}$ & $\begin{array}{c}n=5 \\
4 \pm 0,5 \mathrm{mi} \\
n\end{array}$ & $n=5 t \geq 3 \min$ & $\begin{array}{c}n=5 \\
t \geq 2 \min \end{array}$ & $\begin{array}{c}n=5 \\
t \geq 2 \min \end{array}$ & $\begin{array}{c}n=5 \\
t \geq 2 \min \end{array}$ & $\begin{array}{c}n=5 \\
t \geq 2 \min \end{array}$ & $\begin{array}{c}n=2 \\
38 \pm 5 n=3 \\
t \geq 2 \min \end{array}$ & $\begin{array}{c}n=5 \\
t \geq 2 \min \end{array}$ \\
\hline $\begin{array}{l}\text { Planche à } \\
\text { trous }\end{array}$ & $\begin{array}{l}\text { Trous explorés } \\
\text { au cours de } 5 \\
\text { minutes }\end{array}$ & 7 & 0 & 1 & 0 & 0 & 1 & 2 & 0 \\
\hline
\end{tabular}

Tm1 : temps moyen de chute ; Tm2 : temps moyen de rétablissement

Tableau 5 : Action hypnotique et cataleptique des produits 4a, 4b et 4c

\begin{tabular}{|c|c|c|c|c|c|c|c|c|c|c|c|}
\hline & & \multirow[b]{2}{*}{$\begin{array}{l}\text { Référence } \\
\text { Diazépam }\end{array}$} & \multicolumn{3}{|c|}{$200 \mathrm{mg} / \mathrm{Kg}$} & \multicolumn{3}{|c|}{$400 \mathrm{mg} / \mathrm{Kg}$} & \multicolumn{3}{|c|}{$600 \mathrm{mg} / \mathrm{Kg}$} \\
\hline & & & $4 a$ & $4 b$ & $4 c$ & $4 a$ & $4 b$ & $4 c$ & $4 a$ & $4 b$ & 4c \\
\hline & TE & $7 \pm 1 \min$ & ns & ns & $\mathrm{nc}$ & ns & ns & $1,1 \pm 0,2$ & $5,5 \pm 0,5$ & $4,5 \pm 0,5$ & $6,5 \pm 1$ \\
\hline Hypnotique & TS & $30 \pm 4 \min$ & ns & ns & ns & ns & ns & $5 \pm 1$ & $16 \pm 2$ & $24 \pm 3$ & $30 \pm 3$ \\
\hline Catatonigène & $\mathrm{TC}$ & ns & ns & ns & ns & ns & ns & ns & ns & ns & ns \\
\hline
\end{tabular}

TE : temps moyen d'endormissement en minutes; TS : temps moyen de sommeil en minutes

TC : temps de catalepsie en minutes ; ns : non significatif

Tableau 6 : Interaction médicamenteuse des produits $4 a, 4 b$ et $4 \mathrm{c}$

\begin{tabular}{|c|c|c|c|c|c|c|}
\hline & & $\begin{array}{c}30 \mathrm{mg} / \mathrm{Kg} \\
\text { thio }\end{array}$ & $\begin{array}{c}30 \mathrm{mg} / \mathrm{Kg} \text { thio } \\
\text { et } \\
50 \mathrm{mg} / \mathrm{Kg} \\
\text { diazépam }\end{array}$ & $\begin{array}{c}30 \mathrm{mg} / \mathrm{Kg} \text { thio } \\
\text { et } \\
400 \mathrm{mg} / \mathrm{Kg} 4 \mathrm{a}\end{array}$ & $\begin{array}{c}30 \mathrm{mg} / \mathrm{Kg} \text { thio } \\
\text { et } \\
400 \mathrm{mg} / \mathrm{Kg} \\
4 \mathrm{~b}\end{array}$ & $\begin{array}{c}30 \mathrm{mg} / \mathrm{Kg} \text { thio } \\
\text { et } \\
200 \mathrm{mg} / \mathrm{Kg} 4 \mathrm{c}\end{array}$ \\
\hline $\begin{array}{c}\text { Interaction } \\
\text { Médicamenteuse }\end{array}$ & $\begin{array}{l}\text { TE } \\
\text { TS }\end{array}$ & $\begin{array}{l}\text { Témoin } \\
5 \pm 1 \\
22 \pm 0.5\end{array}$ & $\begin{array}{c}\text { Référence } \\
4 \pm 1 \\
135 \pm 20\end{array}$ & $\begin{array}{c}4 a \\
4,2 \pm 0,2 \\
58 \pm 3\end{array}$ & $\begin{array}{c}4 b \\
4,5 \pm 1 \\
59 \pm 2\end{array}$ & $\begin{array}{c}4 c \\
6 \pm 2 \\
150 \pm 10\end{array}$ \\
\hline
\end{tabular}

TE : temps moyen d'endormissement en minutes ; TS : temps moyen de sommeil en minutes

Thio : thiobarbital 


\section{CONCLUSION}

Les trois produits testés ne sont pas toxiques aux doses expérimentées, et semblent exercer un effet sédatif, hypnotique important sur le système nerveux central. En ce qui concerne le comportement psychomoteur de la souris, les produits s'inscrivent dans l'ordre d'activité décroissante en allant du produit 4c vers les produits $\mathbf{4 a}$ et $\mathbf{4 b}$. En effet le produit $4 \mathrm{c}$ présente une activité supérieure par rapport au produit $\mathbf{4 b}$ qui lui-même semble être plus actif par rapport au produit $4 a$.

\section{REFERENCES}

1. Sotelo E, Ravina E, Estevez I. 1999. The 6aryl-3(2H)-pyridazinones: Synthesis and activity. J. Het. Chem. 36 : 985-990.

2. Taoufik J, Couquelet J.M, Couquelet J.D, Tronche P. 1985. Arylidène-5- méthyl-6-4Hpyridazine et systèmes à noyaux condensés. J. Het. Chem. 22 : 1615-1619.

3. Regragui M. 1993. Synthèse et activité de nouvelles pyridazinones. M. SC. Thèse de troisième cycle, Université Mohamed V, Faculté des sciences de Rabat, 102p

4. Chaard J.L et coll. 1991. Comparative electron impact mass spectra and activity of new 5-benzylidene-4,5-dihydro-(2H)pyridazin-3-one and 4-benzylidene-4,5dihydrooxazin-6-one. Org. Mass. Spect. 26: 1082-1084.

5. Sotelo E, Coelho A, Ravina E. 2003. Pyridazine derivatives still-based approaches in the synthesis of 5-sbstituted-6-phenyl-3( 2H)-pyridazinone. Bull. Chem. Pharm. 51(4) : 427-430.

6. Taoufik $\mathrm{J}$ et coll. 1984. Recherche d'effets sédatifs dans une nouvelle série de pyridazine $=$ Neurosedative effects in a new series of pyridazinones. Ann. Pharm. Fr. 42(2) : 135-144.
7. Chenebit-Rodriguez E. 1973. Action des nucleophiles azotés sur des hydroxy-2 acyl-3 butenolides: études chimiques et pharmacodynamiques, Thèse soutenue le. Université de Clermont-Ferrand, 98p

8. Yamada T, Sukamoto Y.T. 1983. The pyridazine-3-one : synthesis and activity. Eur. J. Med Chem. 18: 209-220.

9. Kerkour M. 1996. Contribution à l'étude de la synthèse et de la réactivité de pyridazinones à propriétés pharmacologiques potentielles. $\mathrm{M}$. SC. Thèse de troisième cycle, Université Mohamed V , Faculté des Sciences de Rabat, 120p.

10. Laroche M-J, Rousselet F. 1990. Les animaux du laboratoire : Ethique et bonnes pratiques. Masson, Paris, 393p

11. Anonyme. (1996). Guide pour les soins et l'utilisation des animaux de aboratoire, Institute of Laboratory Animal Resources, National Academy Press, Washington, D.C $110 p$.

12. Ernest D. et coll. 1993. Manuel sur le soin et l'utilisation des animaux, volume 1

13. Laroche M-J Fabiani P, Rousselet F. 1986. Expertise toxicologique des medicaments, Masson, Paris, 367p.

14. Zellou et coll. 1998. Synthèse et étude pharmacologique des 1,5-benzodiazépines2,4-diones et leurs dérivés alkylés. Ann. Phar. Fr. 56(4) : 169-174.

15. Courvoisier S, Ducrot R. 1990. Psychotropic drugs. In : GARAHINI S, GHETTI v. Elsevier, Amsterdam. 373-391.

16. Zellou A et coll.1998. Synthèse et évaluation pharmacologique des 1,5-benzodiazépine2,4-dithiones et de leurs dérivés alkylés. Ann. Phar. Fr. 56(4) : 175-180. 